\title{
molecules
}

ISSN 1420-3049

www.mdpi.com/journal/molecules

Article

\section{Synthesis and Anticancer Activity of Some New Pyrazolo[3,4-d]pyrimidin-4-one Derivatives}

\author{
Khaled R. A. Abdellatif ${ }^{1}$, Eman K. A. Abdelall ${ }^{1}$, Mohamed A. Abdelgawad ${ }^{1}$, Rasha R. Ahmed ${ }^{2}$ \\ and Rania B. Bakr ${ }^{1, *}$
}

1 Pharmaceutical Organic Chemistry Department, Faculty of Pharmacy, Beni-Suef University, Beni-Suef 62514, Egypt; E-Mails: khaled.ahmed@bsu.edu.eg (K.R.A.A.); Emansherif94@yahoo.com (E.K.A.A.); mhmdgwd@yahoo.com (M.A.A.)

2 Cell Biology and Histology Division, Zoology Department, Faculty of Science, Beni-Suef University, Beni-Suef 62514,Egypt; E-Mail: shorouk2002os@yahoo.com

* Author to whom correspondence should be addressed; E-Mail: raniabakr@ymail.com or rania.mohamed@pharm.bsu.edu.eg; Fax: +2-082-231-7958.

Received: 31 December 2013; in revised form: 27 February 2014 / Accepted: 5 March 2014 / Published: 18 March 2014

\begin{abstract}
Dimethyl-1-phenyl-1H-pyrazolo[3,4- $d][1,3]$ oxazin-4-one (3) was prepared by hydrolysis of ethyl 5-amino-3-methyl-1-phenyl-1H-pyrazole-4-carboxylate (1) to afford the corresponding carboxylic acid $\mathbf{2}$, which was reacted with acetic anhydride to give $\mathbf{3}$. The pyrazolo[3,4- $d][1,3]$ oxazin-4-one 3 was reacted with hydroxylamine hydrochloride, urea, thiourea, thiosemicarbazide, phenylhydrazine and aromatic amines to afford the corresponding pyrazolo[3,4-d] pyrimidin-4-ones 4, 5a,b, 6, 7, 8a-e, respectively. Condensation of pyrazoloxazine derivative 3 with $99 \%$ hydrazine hydrate afforded the 5-aminopyrazolo[3,4- $d$ ] pyrimidine derivative $\mathbf{9}$. Coupling of 9 with aromatic aldehydes yielded a series of 3,6-dimethyl-5-(4-substitutedbenzylideneamino)-1-phenyl-1,5-dihydropyrazolo[3,4- $d]$ pyrimidin4-ones 10a-e. The new compounds were tested for their antitumor activity on the MCF-7 human breast adenocarcinoma cell line. Almost all the tested compounds revealed antitumor activity, especially 3,6-dimethyl-5-(4-nitrobenzylideneamino)-1-phenyl-1,5dihydropyrazolo[3,4- $d]$ pyrimidin-4-one (10e) which displayed the most potent inhibitory activity with a half maximal inhibitory concentration $\left(\mathrm{IC}_{50}\right)$ of $11 \mu \mathrm{M}$.
\end{abstract}

Keywords: pyrazolo[3,4- $d$ ]pyrimidin-4-one; anticancer activity; MCF7 


\section{Introduction}

Cancer remains one of the most life-threatening diseases, taking nearly 7 million lives each year worldwide. It is realized that neither surgery nor radiation nor the two in combination can adequately control metastatic cancer [1], therefore, efforts to cure cancer have been focusing on conventional chemotherapy. However, this type of treatment usually does not discriminate between dividing normal cells and tumor cells, leading to severe side effects [2]. In the last decade, the use of molecular targeted therapies (a new generation of selective cancer drugs which interfere with specific receptors and signaling pathways that promote tumor cell growth) has made treatments more tumor-specific [3].

The chemistry of pyrazolo[3,4- $d]$ pyrimidine derivatives has received great attention due to their structural similarity with purines and hence several pyrazolo[3,4- $d]$ pyrimidine derivatives exhibit promising anticancer activity [4-9]. Different mechanisms account for the cytotoxic effect of this class of compounds, where they had been reported to act as glycogen synthase kinase (GSK) inhibitors [10], cyclin dependent kinase (CDK) inhibitors [11], dual src/Ab1 kinase inhibitors [12] and epidermal growth factor receptor (EGFR) inhibitors [13]. Moreover, many 5-substituted-1-phenyl-1Hpyrazolo[3,4- $d]$ pyrimidin-4-ones were reported to possess antiproliferative activity against breast carcinoma, MCF7 [14,15].

Examples of anticancer drugs currently used in anticancer therapy can be represented by erlotinib (Tarceva $^{\mathrm{TM}}$ ) [16] and gefitinib (Iressa ${ }^{\mathrm{TM}}$ ) [17] which have been approved for the chemotherapeutic treatment of patients with advanced non-small lung cancer. Also, lapatinib (Tykerb ${ }^{\mathrm{TM}}$ ) [18] was approved for the treatment of breast cancer.

In the view of the previous rationale and in continuation of an ongoing program on the synthesis of antitumor compounds [19], in the present study a new series of pyrazolo[3,4- $d$ ]pyrimidin-4-ones has been synthesized and screened in vitro for antitumor activity. The series comprises the derived 5,6-disubstituted pyrazolo[3,4- $d$ ] pyrimidin-4-one pharmacophore that is structurally related to erlotinib and lapatinib (Figure 1). In the present study, the substitution pattern at the 5,6-disubstituted pyrazolo[3,4- $d$ ]pyrimidin-4-one pharmacophore was manipulated so as to create different electronic environments that might affect the lipophilicity and hence the activity of target molecules.

The rationale for the design of target compounds was based upon some structural modifications on the general features of anilinoquinazoline-containing compounds (Figure 1). These modifications comprise a replacement of the benzene moiety in the quinazoline skeleton by a pyrazolo moiety as the pyrazolo moiety is naturally found in the body's purine bases and this is expected to enhance cytotoxic activity. Prompted by these claims, we present a new series of compounds containing 5,6-disubstituted pyrazolo[3,4- $d]$ pyrimidin-4-ones core as anticancer agents. Our strategy is directed toward designing a variety of compounds with diverse chemical properties hypothesizing that the potency of these compounds might be increased by adding alternative binding groups such as a methyl group at position 6, and aroylhydrazone, phenylamino, amide, thioamide, thiosemicarbazide and substituted aryl at position 5 of the pyrazolo[3,4- $d]$ pyrimidine ring. 
Figure 1. Planned design of new pyrazolo[3,4- $d]$ pyrimidine derivatives for cytotoxic activity.

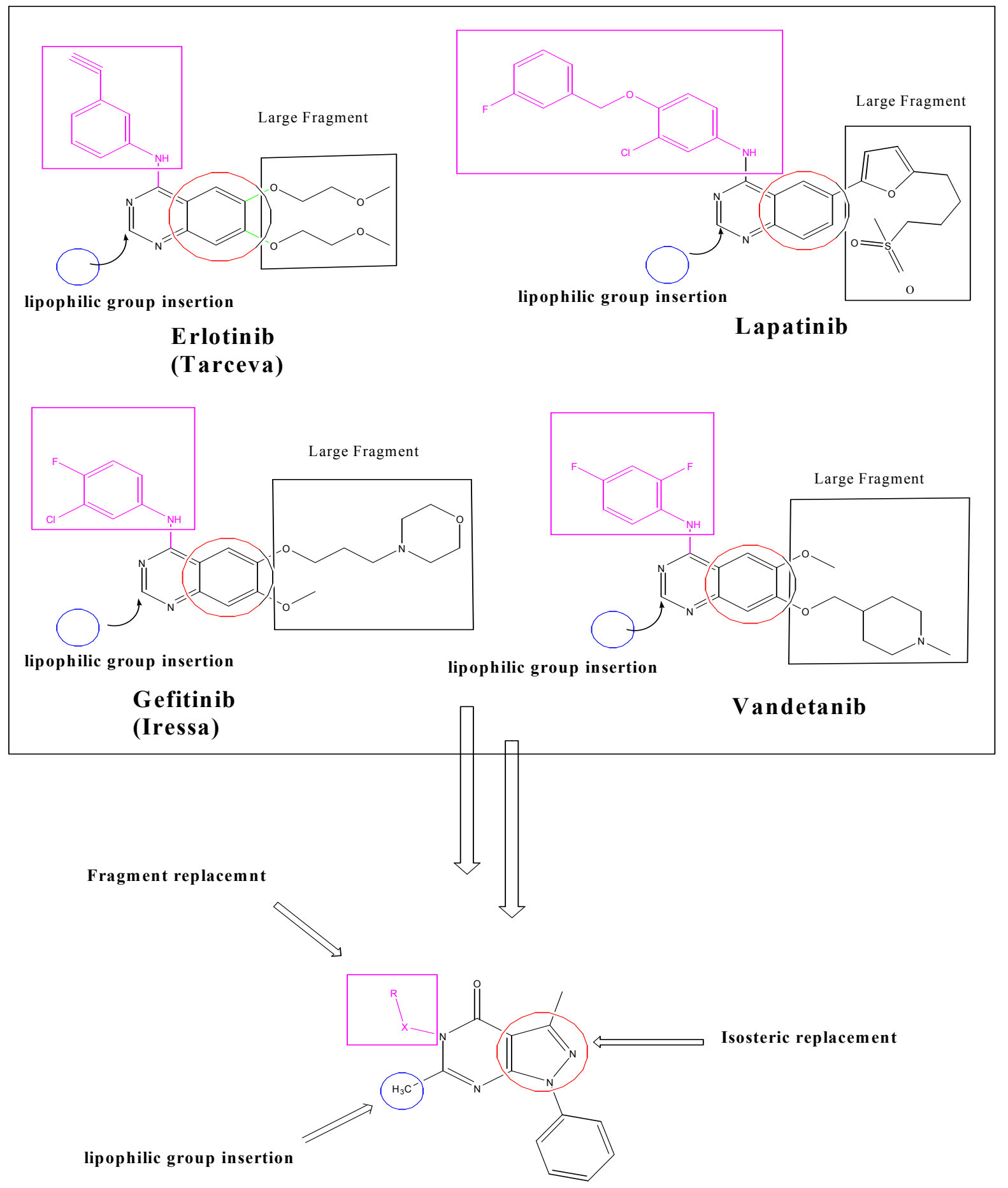

\section{Results and Discussion}

\subsection{Chemical Synthesis}

The synthesis of the target compounds is outlined in Schemes 1 and 2. Accordingly, basic hydrolysis of ethyl 5-amino-3-methyl-1-phenyl-1H-pyrazole-4-carboxylate (1) [20] gave 5-amino-3methyl-1-phenyl-1H-pyrazole-4-carboxylic acid (2) (Scheme 1). The formation of compound 2 was confirmed by ${ }^{1} \mathrm{H}-\mathrm{NMR}$ that showed two $\mathrm{D}_{2} \mathrm{O}$ exchangeable singlet signals at $\delta 6.30,12.08 \mathrm{ppm}$ 
corresponding to $\mathrm{NH}_{2}$ and $\mathrm{COOH}$, respectively. Heating compound 2 with acetic anhydride gave a cyclized product, 3,6-dimethyl-1-phenyl-1H-pyrazolo[3,4- $d][1,3]$ oxazin-4-one (3). The ${ }^{1} \mathrm{H}-\mathrm{NMR}$ spectrum of compound 3 revealed the appearance of a singlet signal at $\delta 2.49 \mathrm{ppm}$ corresponding to the pyrimidine $\mathrm{CH}_{3}$ protons. The mass spectrum of compound 3 showed a molecular ion peak at $\mathrm{m} / \mathrm{z}$ 241 which appeared as the base peak. Reaction of compound 3 with hydroxylamine hydrochloride in dry pyridine afforded 5-hydroxy-3,6-dimethyl-1-phenyl-1,5-dihydropyrazolo[3,4- $d$ ]pyrimidin-4-one (4). The presence of a singlet $\mathrm{OH}$ band at $3,426 \mathrm{~cm}^{-1}$ and $\mathrm{C}=\mathrm{O}$ at $1,680 \mathrm{~cm}^{-1}$ confirmed the formation of 4. Its ${ }^{1} \mathrm{H}-\mathrm{NMR}$ spectrum showed the appearance of a $\mathrm{D}_{2} \mathrm{O}$ exchangeable singlet signal at $\delta 11.52$ ppm corresponding to the $\mathrm{OH}$ proton. The target compounds $\mathbf{5 a}, \mathbf{b}$ and $\mathbf{6}$ were synthesized by fusion at $200{ }^{\circ} \mathrm{C}$ of pyrazoloxazine derivative 3 with urea, thiourea and thiosemicarbazide, respectively. The formation of compounds $\mathbf{5 a}, \mathbf{b}$ and $\mathbf{6}$ was confirmed by their ${ }^{1} \mathrm{H}-\mathrm{NMR}$ spectra that indicated the appearance of exchangeable singlet signals at $\delta 11.15-12.45 \mathrm{ppm}$ corresponding to $\mathrm{NH}$ and $\mathrm{NH}_{2}$. In addition, the mass spectra agreed with the calculated molecular weights of the expected products. On the other hand, reaction of pyrazoloxazine derivative 3 with phenylhydrazine afforded 3,6-dimethyl-1-phenyl-5-phenylamino-1,5-dihydropyrazolo[3,4- $d]$ pyrimidin-4-one (7). The ${ }^{1} \mathrm{H}-\mathrm{NMR}$ of this compound revealed in addition to the corresponding integration for aromatic protons, the presence of NH at $\delta 9.09 \mathrm{ppm}$. The mass spectrum also showed a molecular ion peak at $\mathrm{m} / \mathrm{z} 331$ as the base peak.

Scheme 1. Synthetic pathway for target compounds 4, 5a,b, 6, 7.
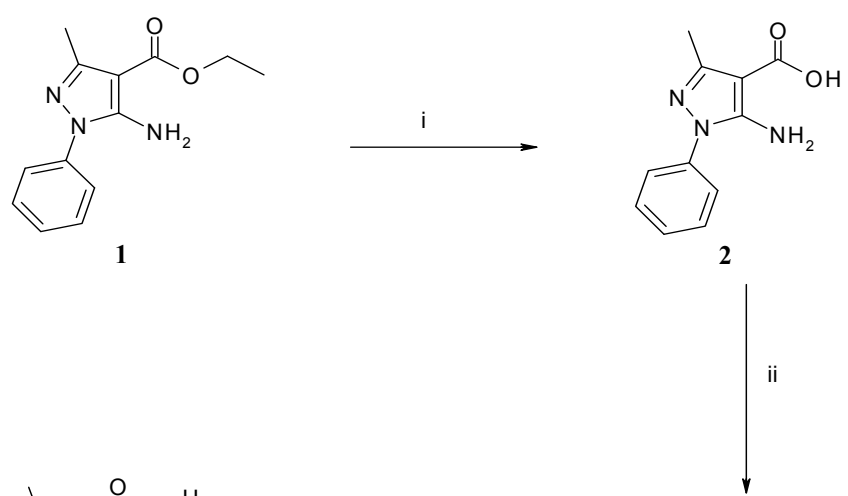

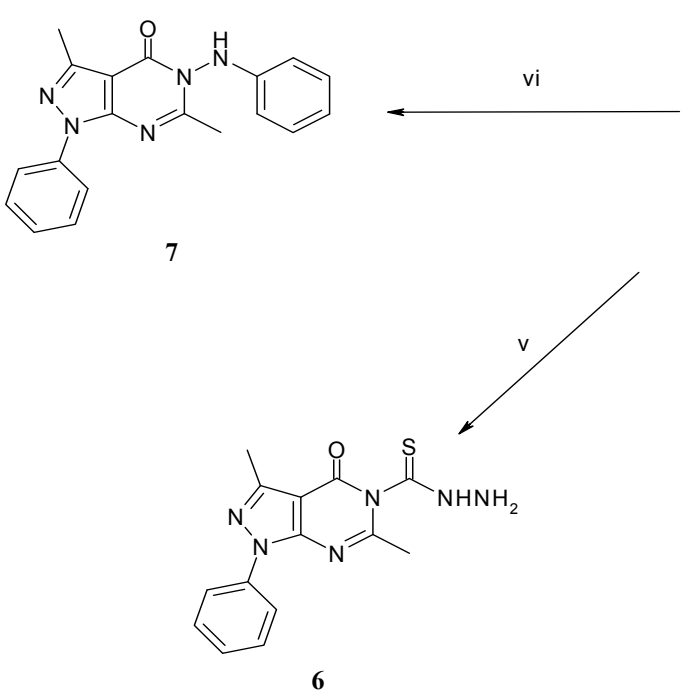

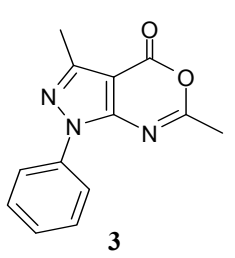
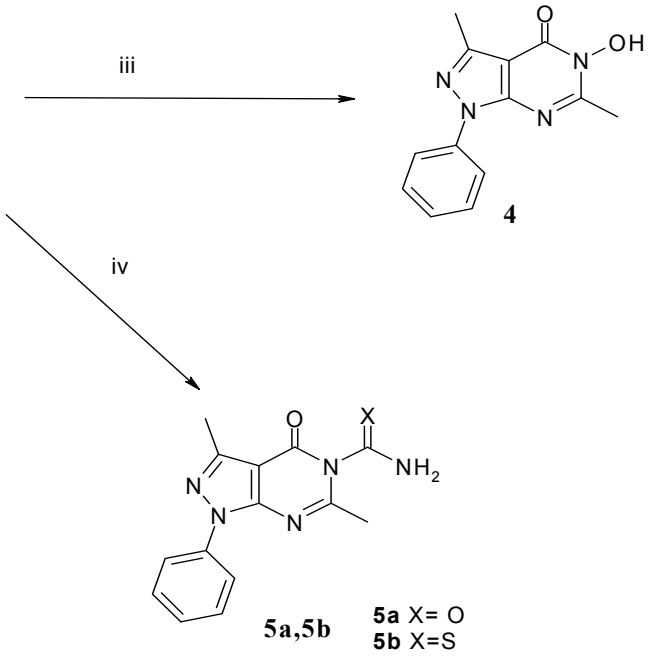

Reagents and conditions: (i) $\mathrm{NaOH}, \mathrm{CH}_{3} \mathrm{OH}$, reflux $5 \mathrm{~h}$; (ii) $\left(\mathrm{CH}_{3} \mathrm{CO}\right)_{2} \mathrm{O}$, reflux $5 \mathrm{~h}$; (iii) $\mathrm{NH}_{2} \mathrm{OH} \cdot \mathrm{HCl}$, dry pyridine, reflux 8h; (iv) $\mathrm{NH}_{2} \mathrm{CXNH}_{2}$, fusion at $200{ }^{\circ} \mathrm{C} 1 \mathrm{~h}$; (v) $\mathrm{NH}_{2} \mathrm{CSNHNH}_{2}$, fusion at $200{ }^{\circ} \mathrm{C} 1 \mathrm{~h}$; (vi) $\mathrm{RC}_{6} \mathrm{H}_{4} \mathrm{NH}_{2}$, dry pyridine, reflux $6 \mathrm{~h}$. 
Scheme 2. Synthetic pathway for target compounds $8 \mathbf{a}-\mathbf{e}$ and $\mathbf{1 0 a}-\mathbf{e}$.

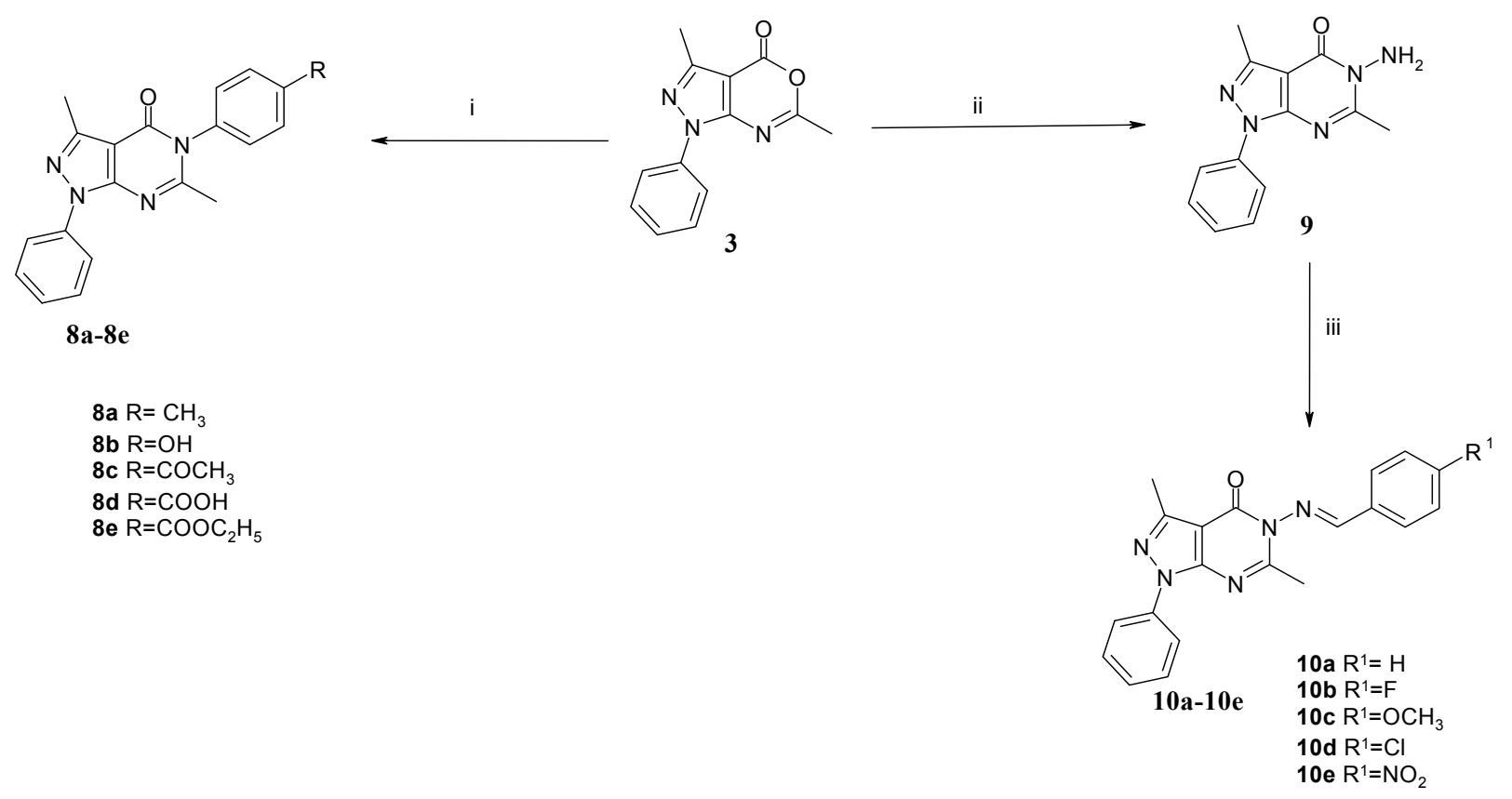

Reagents and conditions: (i) $\mathrm{RC}_{6} \mathrm{H}_{4} \mathrm{NH}_{2}$, dry pyridine, reflux 6 h; (ii) $\mathrm{NH}_{2} \mathrm{NH}_{2} \cdot \mathrm{H}_{2} \mathrm{O}, n$-butanol, reflux $6 \mathrm{~h}$; (iii) $\mathrm{RC}_{6} \mathrm{H}_{4} \mathrm{CHO}$, acetic acid, reflux $20 \mathrm{~h}$.

Moreover, reaction of compound 3 with appropriate aromatic amines furnished 3,6-dimethyl-1phenyl-5-(4-substitutedphenyl)-1,5-dihydropyrazolo[3,4- $d$ ]pyrimidin-4-ones $\mathbf{8 a - e}$ (Scheme 2). The structure of these compounds was established on the basis of their elemental analyses and spectral data. The ${ }^{1} \mathrm{H}-\mathrm{NMR}$ spectra revealed signals at $\delta 6.89-8.15 \mathrm{ppm}$ for aromatic protons. Also, the mass spectra were in agreement with the calculated molecular weights of the synthesized compounds. Condensation of pyrazoloxazine derivative 3 with hydrazine hydrate afforded the 5-amino-pyrazolo[3,4- $d]$ pyrimidin4-one derivative 9. Finally, coupling of 9 with appropriate aromatic aldehydes yielded the corresponding 5-(substituted benzylideneamino)-pyrazolo[3,4- $d]$ pyrimidine derivatives 10a-e. The structures of 10a-e were established on the basis of their elemental analysis and spectral data. The ${ }^{1} \mathrm{H}-\mathrm{NMR}$ spectra of 10a - e showed singlet downfield $\mathrm{CH}=\mathrm{N}$ signals at $\delta 8.32-10.94 \mathrm{ppm}$. It is worth mentioning that the lack of $\mathrm{NH}_{2}$ bands in the IR spectra and $\mathrm{D}_{2} \mathrm{O}$ exchangeable $\mathrm{NH}_{2}$ signals in the ${ }^{1} \mathrm{H}-\mathrm{NMR}$ spectra confirmed the production of the pyrazolo[3,4- $d]$ pyrimidine derivatives $10 a-\mathbf{e}$.

\subsection{In Vitro Anticancer Screening}

The newly synthesized compounds were evaluated for their in vitro cytotoxic activity against human breast cell line (MCF7) using doxorubicin as the reference drug according to the method described as reported by Vichai and Kirtikara [21]. The cytotoxicity was assessed at concentrations of $0,0.01,0.1,10$ and $100 \mu \mathrm{g} / \mathrm{mL}$. The relation between surviving fraction and drug concentration was plotted to obtain the survival curve of MCF7 tumor cell line after addition of the specified compound. The parameter used here is $\mathrm{IC}_{50}$, which corresponds to the concentration required for $50 \%$ inhibition of cell viability. $\mathrm{The} \mathrm{IC}_{50}$ values of the synthesized compounds compared to the reference drug are shown in Table 1. 
Table 1. Results of in vitro cytotoxic activity of the synthesized compounds on human breast cancer cell line (MCF7).

\begin{tabular}{cc}
\hline Compound No. & IC $_{\mathbf{5 0}}$ in $\boldsymbol{\mu M}$ \\
\hline Doxorubicin & 5 \\
$\mathbf{4}$ & 49 \\
$\mathbf{5 a}$ & 52 \\
$\mathbf{5 b}$ & 38 \\
$\mathbf{6}$ & 52 \\
$\mathbf{7}$ & 14 \\
$\mathbf{8 a}$ & 33 \\
$\mathbf{8 b}$ & 25 \\
$\mathbf{8 c}$ & 26 \\
$\mathbf{8 d}$ & 25 \\
$\mathbf{8 e}$ & 27 \\
$\mathbf{9}$ & 84 \\
$\mathbf{1 0 a}$ & 17 \\
$\mathbf{1 0 b}$ & 12 \\
$\mathbf{1 0 c}$ & 18 \\
$\mathbf{1 0 d}$ & 12 \\
$\mathbf{1 0 e}$ & 11 \\
\hline
\end{tabular}

The obtained data revealed that most of the newly synthesized compounds showed potent antitumor activity. Among the tested compounds, the most potent cytotoxic effect against MCF-7 cell line was obtained with the compound 5-(4-nitrobenzylideneamino)pyrazolo[3,4- $d$ ]pyrimidin-4-one (10e) with an $\mathrm{IC}_{50}$ value of $11 \mu \mathrm{M}$, followed by $\mathbf{1 0 d}$ which showed an $\mathrm{IC}_{50}$ value of $12 \mu \mathrm{M}$. Compound 9 exhibited the least cytotoxic activity.

From the antitumor screening results the against the MCF-7 cell line, some structure activity relationships can be suggested. Aromatic substitution on N5 position favors the activity; this is obvious upon comparing 5-(4-hydroxyphenylpyrazolo[3,4- $d$ ]pyrimidin-4-one derivative $\mathbf{8 b}\left(\mathrm{IC}_{50}=25\right)$ and 5-hydroxy-3,6-dimethyl-1-phenyl-1,5-dihydropyrazolo[3,4- $d]$ pyrimidin-4-one (4) $\left(\mathrm{IC}_{50}=49\right)$. Also, the introduction of a 5-amino function on the pyrazolo[3,4- $d]$ pyrimidine core produced inactive compound $9\left(\mathrm{IC}_{50}=84\right)$, while the introduction of an anilino function favors the anticancer activity as shown by compound $7\left(\mathrm{IC}_{50}=14\right)$. Conversion of inactive compound 9 into a 5-substituted benzylidene amino function yielded compounds $\mathbf{1 0 a}-\mathbf{e}$, the most active members of this study. The influence of substituents on the benzylideneamino group on the antitumor activity was in the order $\mathrm{NO}_{2}>\mathrm{F}>\mathrm{Cl}>$ $\mathrm{OCH}_{3}>\mathrm{H}$. Moreover, 5-(4-substituted benzylideneamino) derivatives 10a-e with azomethine spacer groups were more potent than the 5-(4-substituted phenyl) derivatives $\mathbf{8 a - e}$.

\section{Experimental}

\subsection{General}

Melting points were determined on a Griffin apparatus and are uncorrected. IR spectra were determined on Shimadzu IR 435 spectrophotometer and values are presented in $\mathrm{cm}^{-1}$. ${ }^{1} \mathrm{H}-\mathrm{NMR}$ spectra 
were recorded on Varian Gemini $300 \mathrm{MHz}$ spectrometer, using TMS as internal standard and chemical shifts are reported in ppm on the $\delta$ scale. The electron impact (EI) mass spectra were recorded on a Shimadzu QP-2010 plus instrument. Analytical thin layer chromatography (TLC) on silica gel plates containing UV indicator was routinely employed to follow the course of reactions and to check the purity of products. All reagents and solvents were purified and dried by standard techniques. Elemental microanalyses were carried out at the Microanalytical Center, Cairo University.

5-Amino-3-methyl-1-phenyl-1H-pyrazole-4-carboxylic acid (2). A mixture of ethyl 5-amino-3-methyl1-phenyl-1H-pyrazole-4-carboxylate $(1,12.25 \mathrm{~g}, 50 \mathrm{mmol})$ and sodium hydroxide $(4.20 \mathrm{~g}, 10 \mathrm{mmol})$ in methanol $(60 \mathrm{~mL})$ was heated under reflux for $5 \mathrm{~h}$. After cooling, the reaction mixture was poured into ice-cold water, then adjusting $\mathrm{pH}$ of the mixture to 4 using concentrated hydrochloric acid. The solid obtained was filtered, dried and crystallized from ethanol/water. Mp 156-157 ${ }^{\circ} \mathrm{C}$; yield: $56 \%$; IR ( $\left.\mathrm{cm}^{-1}\right)$ : 3389-3204 (OH \& $\left.\mathrm{NH}_{2}\right) ; 3009(\mathrm{CH}$ aromatic); $2927(\mathrm{CH}$ aliphatic); $1651(\mathrm{C}=\mathrm{O}) ; 1613(\mathrm{C}=\mathrm{N})$; ${ }^{1} \mathrm{H}-\mathrm{NMR}\left(\mathrm{DMSO}-d_{6}\right) \delta$ ppm: 2.25 (s, 3H); $6.30(\mathrm{~s}, 2 \mathrm{H}) ; 7.36-7.54(\mathrm{~m}, 5 \mathrm{H}) ; 12.08(\mathrm{~s}, 1 \mathrm{H})$; MS $m / z$ : $217\left(\mathrm{M}^{+}, 27.65\right)$;. Anal. Calcd for $\mathrm{C}_{11} \mathrm{H}_{11} \mathrm{~N}_{3} \mathrm{O}_{2}$ (217.23): C, 60.82; H, 5.10; N, 19.34. Found: C, 60.69; H, 5.20; N, 19.67.

3,6-Dimethyl-1-phenyl-1H-pyrazolo[3,4-d][1,3]oxazin-4-one (3). A mixture of 5-amino-3-methyl-1phenyl-1H-pyrazole-4-carboxylic acid $(2,2.17 \mathrm{~g}, 10 \mathrm{mmol})$ and acetic anhydride $(5 \mathrm{~mL})$ was heated under reflux for $5 \mathrm{~h}$. After cooling, the formed solid was filtered, dried and crystallized from methanol. Mp 129-130 ${ }^{\circ} \mathrm{C}$; yield: 49\%; IR ( $\left.\mathrm{cm}^{-1}\right)$ : 3078 (CH aromatic); 2923 (CH aliphatic); $1764(\mathrm{C}=\mathrm{O}) ; 1599$ $(\mathrm{C}=\mathrm{N}) ;{ }^{1} \mathrm{H}-\mathrm{NMR}\left(\mathrm{DMSO}-d_{6}\right) \delta \mathrm{ppm}: 2.46(\mathrm{~s}, 3 \mathrm{H}) ; 2.49(\mathrm{~s}, 3 \mathrm{H}) ; 7.43(\mathrm{t}, J=9 \mathrm{~Hz}, 1 \mathrm{H}) ; 7.57(\mathrm{t}, J=9 \mathrm{~Hz}$, $2 \mathrm{H}) ; 7.90(\mathrm{~d}, J=9 \mathrm{~Hz}, 2 \mathrm{H})$; $\mathrm{MS} m / z: 241\left(\mathrm{M}^{+}, 100\right)$. Anal. Calcd for $\mathrm{C}_{13} \mathrm{H}_{11} \mathrm{~N}_{3} \mathrm{O}_{2}$ (241.25): $\mathrm{C}, 64.72 ; \mathrm{H}$, 4.60; N, 17.42. Found: C, 65.01; H, 4.73; N, 17.19 .

3,6-Dimethyl-5-hydroxy-1-phenyl-1,5-dihydropyrazolo[3,4-d]pyrimidin-4-one (4). A mixture of compound 3 (2.41 g, $10 \mathrm{mmol})$ and hydroxylamine hydrochloride $(0.69 \mathrm{~g}, 10 \mathrm{mmol})$ in dry pyridine $(30 \mathrm{~mL})$ was heated under reflux for $8 \mathrm{~h}$. The reaction mixture was concentrated to its half volume and the separated solid was filtered, washed with water, dried and crystallized from dioxane. Mp $180-181{ }^{\circ} \mathrm{C}$; yield: 74\%; IR ( $\left.\mathrm{cm}^{-1}\right)$ : $3426(\mathrm{OH}) ; 2926\left(\mathrm{CH}\right.$ aliphatic); $1680(\mathrm{C}=\mathrm{O}) ; 1568(\mathrm{C}=\mathrm{N}) ;{ }^{1} \mathrm{H}-\mathrm{NMR}\left(\mathrm{DMSO}-d_{6}\right)$ $\delta$ ppm: 2.48 (s, 3H); $2.54(\mathrm{~s}, 3 \mathrm{H}) ; 7.35(\mathrm{t}, J=8.4 \mathrm{~Hz}, 1 \mathrm{H}) ; 7.53(\mathrm{t}, J=8.4 \mathrm{~Hz}, 2 \mathrm{H}) ; 8.03(\mathrm{~d}, J=9 \mathrm{~Hz}$, $2 \mathrm{H}) ; 11.52(\mathrm{~s}, 1 \mathrm{H}) ;{ }^{13} \mathrm{C}-\mathrm{NMR}$ (DMSO-d $d_{6} \delta \mathrm{ppm}: 13.71,21.38,105.07,121.58,126.92,129.59$, 138.75, 145.98, 149.94, 155.24, 157.86. MS m/z: $256\left(\mathrm{M}^{+}, 89.42\right)$; Anal. Calcd for $\mathrm{C}_{13} \mathrm{H}_{12} \mathrm{~N}_{4} \mathrm{O}_{2}$ (256.27): C, 60.93; H, 4.72; N, 21.86. Found: C, 60.81; H, 5.00; N, 21.66.

3.1.1. General Procedure for the Synthesis of 3,6-Dimethyl-1-phenyl-5-substituted-1,5-dihydropyrazolo[3,4- $d]$ pyrimidin-4-ones 5a,b

A mixture of 3,6-dimethyl-1-phenyl-1H-pyrazolo[3,4- $d][1,3]$ oxazin-4-one $(3,2.41 \mathrm{~g}, 10 \mathrm{mmol})$ and urea or thiourea $(10 \mathrm{mmol})$ was fused at $200{ }^{\circ} \mathrm{C}$ for $1 \mathrm{~h}$. The mixture was cooled and methanol was added to the reaction mixture. The separated solid was filtered, washed with methanol, dried and crystallized from ethanol. 
3,6-Dimethyl-4-oxo-1-phenyl-1,4-dihydropyrazolo[3,4-d]pyrimidine-5-carboxamide (5a). $\mathrm{Mp}>300{ }^{\circ} \mathrm{C}$; yield: 65\%; IR ( $\left.\mathrm{cm}^{-1}\right)$ : 3432-3344 $\left(\mathrm{NH}_{2}\right) ; 3073$ (CH aromatic); 2926 (CH aliphatic); 1671 (broad band of $2 \mathrm{C}=\mathrm{O}) ; 1592(\mathrm{C}=\mathrm{N}) ;{ }^{1} \mathrm{H}-\mathrm{NMR}\left(\mathrm{DMSO}-d_{6}\right) \delta \mathrm{ppm}: 2.35(\mathrm{~s}, 3 \mathrm{H}) ; 2.46(\mathrm{~s}, 3 \mathrm{H}) ; 7.34(\mathrm{t}, J=7.5 \mathrm{~Hz}$, $1 \mathrm{H}) ; 7.51(\mathrm{t}, J=7.5 \mathrm{~Hz}, 2 \mathrm{H}) ; 8.02(\mathrm{~d}, J=9 \mathrm{~Hz}, 2 \mathrm{H}) ; 12.20$ (s, 2H); MS m/z: $283\left(\mathrm{M}^{+}, 12.81\right)$; Anal. Calcd for $\mathrm{C}_{14} \mathrm{H}_{13} \mathrm{~N}_{5} \mathrm{O}_{2}$ (283.29): C, 59.36; H, 4.63; N, 24.72. Found: C, 59.55; H, 4.52; N, 24.94.

3,6-Dimethyl-4-oxo-1-phenyl-1,4-dihydropyrazolo[3,4-d]pyrimidine-5-carbothioamide (5b). $\mathrm{Mp}>300{ }^{\circ} \mathrm{C}$; yield: 45\%; IR ( $\left.\mathrm{cm}^{-1}\right)$ : $3425\left(\mathrm{NH}_{2}\right) ; 2923(\mathrm{CH}$ aliphatic); $1675(\mathrm{C}=\mathrm{O}) ; 1596(\mathrm{C}=\mathrm{N}) ; 1118(\mathrm{C}=\mathrm{S})$; ${ }^{1} \mathrm{H}-\mathrm{NMR}\left(\mathrm{DMSO}-d_{6}\right) \delta \mathrm{ppm}: 2.38(\mathrm{~s}, 3 \mathrm{H}) ; 2.48(\mathrm{~s}, 3 \mathrm{H}) ; 7.35(\mathrm{t}, J=7.2 \mathrm{~Hz}, 1 \mathrm{H}) ; 7.51$ (t, $J=7.2 \mathrm{~Hz}$, $2 \mathrm{H}) ; 8.02(\mathrm{~d}, J=9 \mathrm{~Hz}, 2 \mathrm{H}) ; 12.45(\mathrm{~s}, 2 \mathrm{H})$; Anal. Calcd for $\mathrm{C}_{14} \mathrm{H}_{13} \mathrm{~N}_{5} \mathrm{OS}$ (299.36): C, 56.17; H, 4.38; $\mathrm{N}, 23.39$. Found: C, 56.22; H, 4.21; N, 23.64.

3,6-Dimethyl-4-oxo-1-phenyl-1,4-dihydropyrazolo[3,4-d]pyrimidine-5-carbothioic acid hydrazide (6). A mixture of 3,6-dimethyl-1-phenyl-1H-pyrazolo[3,4- $d][1,3]$ oxazin-4-one (3, $2.41 \mathrm{gm}, 10 \mathrm{mmol})$ and thiosemicarbazide $(0.91 \mathrm{~g}, 10 \mathrm{mmol})$ was fused at $200{ }^{\circ} \mathrm{C}$ for $1 \mathrm{~h}$. The reaction mixture was cooled and methanol was added to the reaction mixture. The separated solid was filtered, washed with methanol, dried and crystallized from acetone. $\mathrm{Mp}>250-251^{\circ} \mathrm{C}$; yield: $63 \%$; IR $\left(\mathrm{cm}^{-1}\right)$ : 3321 (broad band of $\left.\mathrm{NH} \& \mathrm{NH}_{2}\right) ; 3064(\mathrm{CH}$ aromatic); $2920(\mathrm{CH}$ aliphatic); $1694(\mathrm{C}=\mathrm{O}) ; 1590(\mathrm{C}=\mathrm{N}) ; 1105(\mathrm{C}=\mathrm{S})$; ${ }^{1} \mathrm{H}-\mathrm{NMR}\left(\mathrm{DMSO}-d_{6}\right) \delta$ ppm: $2.37(\mathrm{~s}, 3 \mathrm{H}) ; 2.49(\mathrm{~s}, 3 \mathrm{H}) ; 7.35(\mathrm{t}, J=6 \mathrm{~Hz}, 1 \mathrm{H}) ; 7.51(\mathrm{t}, J=7.2 \mathrm{~Hz}, 2 \mathrm{H})$; $8.01(\mathrm{~d}, J=9 \mathrm{~Hz}, 2 \mathrm{H}) ; 11.15(\mathrm{~s}, 1 \mathrm{H}) ; 12.19(\mathrm{~s}, 2 \mathrm{H})$; MS m/z: $314\left(\mathrm{M}^{+}, 6.92\right)$; Anal. Calcd for $\mathrm{C}_{14} \mathrm{H}_{14} \mathrm{~N}_{6} \mathrm{OS}$ (314.37): C, 53.49; H, 4.49; N, 26.73. Found: C, 53.28; H, 4.47; N, 26.89.

3,6-Dimethyl-1-phenyl-5-phenylamino-1,5-dihydropyrazolo[3,4-d]pyrimidin-4-one (7). A mixture of 3,6-dimethyl-1-phenyl-1H-pyrazolo[3,4-d][1,3]oxazin-4-one (3, $2.41 \mathrm{~g}, 10 \mathrm{mmol})$ and phenylhydrazine (1.08 gm, $10 \mathrm{mmol})$ in ethanol $(20 \mathrm{~mL})$ was heated under reflux for $6 \mathrm{~h}$. The separated solid was filtered while hot, dried and crystallized from butanol. Mp $265-266{ }^{\circ} \mathrm{C}$; yield: $54 \%$; IR $\left(\mathrm{cm}^{-1}\right): 3250$ $(\mathrm{NH}) ; 3115-3011$ (CH aromatic); 2930 (CH aliphatic); $1685(\mathrm{C}=\mathrm{O}) ;{ }^{1} \mathrm{H}-\mathrm{NMR}$ (DMSO- $d_{6}$ ) $\delta$ ppm: 2.49 $(\mathrm{s}, 3 \mathrm{H}) ; 2.53(\mathrm{~s}, 3 \mathrm{H}) ; 6.69(\mathrm{~d}, J=7.2 \mathrm{~Hz}, 2 \mathrm{H}) ; 6.86(\mathrm{t}, J=7.5 \mathrm{~Hz}, 1 \mathrm{H}) ; 7.21(\mathrm{t}, J=7.5 \mathrm{~Hz}, 2 \mathrm{H}) ; 7.39$ (t, $J=7.8 \mathrm{~Hz}, 1 \mathrm{H}) ; 7.56$ (t, $J=7.8 \mathrm{~Hz}, 2 \mathrm{H}) ; 8.05$ (d, $J=9 \mathrm{~Hz}, 2 \mathrm{H}) ; 9.09$ (s, 1H); MS m/z: $331\left(\mathrm{M}^{+}, 100\right)$; Anal. Calcd for $\mathrm{C}_{19} \mathrm{H}_{17} \mathrm{~N}_{5} \mathrm{O}$ (331.38): C, 68.87; H, 5.17; N, 21.13. Found: C68.68; H, 5.09; N, 21.05.

3.1.2. General Procedure for the Synthesis of 3,6-Dimethyl-1-phenyl-5-(4-substitutedphenyl)-1,5dihydropyrazolo[3,4- $d]$ pyrimidin-4-ones 8a-e

A mixture of 3,6-dimethyl-1-phenyl-1H-pyrazolo[3,4- $d][1,3]$ oxazin-4-one $(3,2.41 \mathrm{~g}, 10 \mathrm{mmol})$ and the appropriate aromatic amine $(10 \mathrm{mmol})$ in dry pyridine $(30 \mathrm{~mL})$ was heated under reflux for $6 \mathrm{~h}$. The reaction mixture was poured into ice-cold water and the mixture was neutralized with hydrochloric acid (10\%). The separated product was filtered, washed with water, dried and crystallized from the appropriate solvent.

3,6-Dimethyl-1-phenyl-5-p-tolyl-1,5-dihydropyrazolo[3,4-d]pyrimidin-4-one (8a). Mp 170-171 ${ }^{\circ} \mathrm{C}$ (crystallized from methanol); yield: 50\%; IR $\left(\mathrm{cm}^{-1}\right)$ : $2923(\mathrm{CH}$ aliphatic); $1693(\mathrm{C}=\mathrm{O}) ; 1568(\mathrm{C}=\mathrm{N})$; ${ }^{1} \mathrm{H}-\mathrm{NMR}\left(\mathrm{DMSO}-d_{6}\right) \delta$ ppm: $2.16(\mathrm{~s}, 3 \mathrm{H}) ; 2.51(\mathrm{~s}, 3 \mathrm{H}) ; 2.66(\mathrm{~s}, 3 \mathrm{H}) ; 7.38-7.41(\mathrm{~m}, 1 \mathrm{H}) ; 7.56-7.62$ 
(m, 4H); 8.07-8.15 (m, 4H); MS m/z: $330\left(\mathrm{M}^{+}, 100\right)$; Anal. Calcd for $\mathrm{C}_{20} \mathrm{H}_{18} \mathrm{~N}_{4} \mathrm{O}$ (330.39): C, 72.71; H, 5.49; N, 16.96. Found: C, 72.77; H, 5.20; N, 16.64.

3,6-Dimethyl-5-(4-hydroxyphenyl)-1-phenyl-1,5-dihydropyrazolo[3,4-d]pyrimidin-4-one $\quad(\mathbf{8 b}) . \quad \mathrm{Mp}$ 250-252 ${ }^{\circ} \mathrm{C}$ (crystallized from acetone); yield: 76\%; IR ( $\left.\mathrm{cm}^{-1}\right)$ : $3439(\mathrm{OH}) ; 2930$ (CH aliphatic); 1694 $(\mathrm{C}=\mathrm{O}) ; 1566(\mathrm{C}=\mathrm{N}) ;{ }^{1} \mathrm{H}-\mathrm{NMR}\left(\mathrm{DMSO}_{-}\right) \delta \mathrm{ppm}: 2.17(\mathrm{~s}, 3 \mathrm{H}) ; 2.51(\mathrm{~s}, 3 \mathrm{H}) ; 6.89(\mathrm{~d}, J=9 \mathrm{~Hz}, 2 \mathrm{H})$; $7.17(\mathrm{~d}, J=9 \mathrm{~Hz}, 2 \mathrm{H}) ; 7.38(\mathrm{t}, J=7.5 \mathrm{~Hz}, 1 \mathrm{H}) ; 7.53(\mathrm{t}, J=7.5 \mathrm{~Hz}, 1 \mathrm{H}) ; 8.07$ (d, $J=9 \mathrm{~Hz}, 2 \mathrm{H}) ; 9.80$ (s, IH); MS m/z: $332\left(\mathrm{M}^{+}, 100\right)$; Anal. Calcd for $\mathrm{C}_{19} \mathrm{H}_{16} \mathrm{~N}_{4} \mathrm{O}_{2}$ (332.36): C, 68.66; H, 4.85; N, 16.86 . Found: C, 68.30; H, 4.50; N, 16.98.

5-(4-Acetylphenyl)-3,6-dimethyl-1-phenyl-1,5-dihydropyrazolo[3,4-d]pyrimidin-4-one (8c). Mp 186-187 ${ }^{\circ} \mathrm{C}$ (crystallized from ethanol); yield: 66\%; IR $\left(\mathrm{cm}^{-1}\right)$ : 2924 ( $\mathrm{CH}$ aliphatic); 1699 (broad band of 2C=O); $1591(\mathrm{C}=\mathrm{N}) ;{ }^{1} \mathrm{H}-\mathrm{NMR}\left(\mathrm{DMSO}-d_{6}\right) \delta \mathrm{ppm}: 2.17(\mathrm{~s}, 3 \mathrm{H}) ; 2.44(\mathrm{~s}, 3 \mathrm{H}) ; 2.50(\mathrm{~s}, 3 \mathrm{H}) ; 7.23(\mathrm{~d}, J=9 \mathrm{~Hz}$, 2H); $7.26(\mathrm{~d}, J=9 \mathrm{~Hz}, 2 \mathrm{H}) ; 7.52-7.64(\mathrm{~m}, 3 \mathrm{H}) ; 8.08$ (d, $J=9 \mathrm{~Hz}, 2 \mathrm{H}) ; \mathrm{MS} m / z: 358\left(\mathrm{M}^{+}, 100\right)$; Anal. Calcd for $\mathrm{C}_{21} \mathrm{H}_{18} \mathrm{~N}_{4} \mathrm{O}_{2}$ (358.40): C, 70.38; H, 5.06; N, 15.63. Found: C, 69.97; H, 4.98; N, 15.94 .

4-(3,6-Dimethyl-4-oxo-1-phenyl-1,5-dihydropyrazolo[3,4-d]pyrimidin-5-yl)benzoic acid (8d). Mp 200-201 ${ }^{\circ} \mathrm{C}$ (crystallized from acetic acid); yield: 59\%; IR $\left(\mathrm{cm}^{-1}\right): 3450(\mathrm{OH}) ; 3068(\mathrm{CH}$ aromatic); 2929 (CH aliphatic); 1713, $1678(2 \mathrm{C}=\mathrm{O}) ; 1558(\mathrm{C}=\mathrm{N}) ;{ }^{1} \mathrm{H}$ NMR (DMSO- $\left.d_{6}\right) \delta$ ppm: $2.16(\mathrm{~s}, 3 \mathrm{H})$; $2.51(\mathrm{~s}, 3 \mathrm{H}) ; 7.38$ (t, $J=7.2 \mathrm{~Hz}, 1 \mathrm{H}) ; 7.54-7.65(\mathrm{~m}, 4 \mathrm{H}) ; 8.07-8.13(\mathrm{~m}, 4 \mathrm{H}) ; 13.15$ (s, IH); MS $m / z$ : $360\left(\mathrm{M}^{+}\right.$, 79.41); Anal. Calcd for $\mathrm{C}_{20} \mathrm{H}_{16} \mathrm{~N}_{4} \mathrm{O}_{3}$ (360.38): C, 66.66; H, 4.48; N, 15.55. Found: C, 66.90; $\mathrm{H}, 4.71 ; \mathrm{N}, 15.78$.

Ethyl 4-(3,6-dimethyl-4-oxo-1-phenyl-1,5-dihydropyrazolo[3,4-d]pyrimidin-5-yl)benzoate (8e). Mp 190-191 ${ }^{\circ} \mathrm{C}$ (crystallized from ethanol); yield: 85\%; IR ( $\left.\mathrm{cm}^{-1}\right)$ : 2925 (CH aliphatic); 1709 (broad band of $2 \mathrm{C}=\mathrm{O}) ; 1555(\mathrm{C}=\mathrm{N}) ;{ }^{1} \mathrm{H}$ NMR (DMSO- $\left.d_{6}\right) \delta \mathrm{ppm}$ : $1.36(\mathrm{t}, J=7.2 \mathrm{~Hz}, 3 \mathrm{H}) ; 2.15(\mathrm{~s}, 3 \mathrm{H})$; 2.51 (s, 3H); 4.37 (q, $J=7.2 \mathrm{~Hz}, 2 \mathrm{H}) ; 7.39$ (t, $J=7.2 \mathrm{~Hz}, 1 \mathrm{H}) ; 7.53-7.62$ (m, 4H); 8.07-8.15 (m, 4H); MS m/z: $388\left(\mathrm{M}^{+}, 93.03\right)$; Anal. Calcd for $\mathrm{C}_{22} \mathrm{H}_{20} \mathrm{~N}_{4} \mathrm{O}_{3}$ (388.43): C, 68.03; H, 5.19; N, 14.42. Found: C $68.21 ; \mathrm{H}, 5.51 ; \mathrm{N}, 14.66$.

5-Amino-3,6-dimethyl-1-phenyl-1,5-dihydropyrazolo[3,4-d]pyrimidin-4-one (9). A mixture of compound 3 (2.41 g, $10 \mathrm{mmol})$ and hydrazine hydrate $(0.5 \mathrm{~mL}, 10 \mathrm{mmol})$ in butanol $(20 \mathrm{~mL})$ was heated under reflux for $6 \mathrm{~h}$. After cooling, the separated solid was filtered, dried and crystallized from ethanol. Mp 129-130 ${ }^{\circ} \mathrm{C}$; yield: 47\%; IR $\left(\mathrm{cm}^{-1}\right)$ : 3322-3262 (forked, $\mathrm{NH}_{2}$ ); 3065 (CH aromatic); 2924 (CH aliphatic); $1692(\mathrm{C}=\mathrm{O}) ;{ }^{1} \mathrm{H}-\mathrm{NMR}\left(\mathrm{DMSO}-d_{6}\right) \delta \mathrm{ppm}: 2.50(\mathrm{~s}, 3 \mathrm{H}) ; 2.59(\mathrm{~s}, 3 \mathrm{H}) ; 5.71(\mathrm{~s}, 2 \mathrm{H}) ; 7.34$ (t, $J=7.5 \mathrm{~Hz}, 1 \mathrm{H}) ; 7.52$ (t, $J=7.5 \mathrm{~Hz}, 2 \mathrm{H}) ; 8.05(\mathrm{~d}, J=9 \mathrm{~Hz}, 2 \mathrm{H})$; MS $m / z: 255\left(\mathrm{M}^{+}, 100\right)$. Anal. Calcd for $\mathrm{C}_{13} \mathrm{H}_{13} \mathrm{~N}_{5} \mathrm{O}$ (255.28): C, 61.17; H, 5.13; N, 27.43. Found: C, 61.53; H, 5.03; N, 27.23.

3.1.3. General Procedure for the Synthesis of (E)-3,6-Dimethyl-5-(4-substitutedbenzylideneamino)-1phenyl-1,5-dihydropyrazolo[3,4- $d]$ pyrimidin-4-ones 10a-e

A mixture of compound 9 (2.55 gm, $10 \mathrm{mmol})$ and the appropriate aromatic aldehyde (12 mmol) in acetic acid $(15 \mathrm{~mL})$ was heated under reflux for $20 \mathrm{~h}$. The reaction mixture was concentrated under 
vacuum then cooled. The obtained solid was filtered, washed with water, dried and crystallized from the appropriate solvent. The spectral data for compounds $\mathbf{1 0 a}-\mathbf{e}$ are listed below.

(E)-5-(Benzylideneamino)-3,6-dimethyl-1-phenyl-1,5-dihydropyrazolo[3,4-d]pyrimidin-4-one (10a). Mp 250-251 ${ }^{\circ} \mathrm{C}$ (crystallized from ethanol); yield: 70\%; IR $\left(\mathrm{cm}^{-1}\right): 3055(\mathrm{CH}$ aromatic); $2925(\mathrm{CH}$ aliphatic); $1702(\mathrm{C}=\mathrm{O}) ; 1555(\mathrm{C}=\mathrm{N}) ;{ }^{1} \mathrm{H}-\mathrm{NMR}\left(\mathrm{DMSO}-d_{6}\right) \delta$ ppm: $2.50(\mathrm{~s}, 3 \mathrm{H}) ; 2.55(\mathrm{~s}, 3 \mathrm{H}) ; 7.39$ (t, $J=9 \mathrm{~Hz}, 1 \mathrm{H}) ; 7.53-7.65(\mathrm{~m}, 5 \mathrm{H}) ; 7.98(\mathrm{~d}, J=9 \mathrm{~Hz}, 2 \mathrm{H}) ; 8.08(\mathrm{~d}, J=9 \mathrm{~Hz}, 2 \mathrm{H}) ; 8.89$ (s, 1H); MS m/z: $343\left(\mathrm{M}^{+}, 4.62\right)$; Anal. Calcd for $\mathrm{C}_{20} \mathrm{H}_{17} \mathrm{~N}_{5} \mathrm{O}$ (343.39): C, 69.96; H, 4.99; N, 20.39. Found: C, 69.77; H, 5.20; N, 20.54 .

(E)-3,6-Dimethyl-5-(4-fluorobenzylideneamino)-1-phenyl-1,5-dihydropyrazolo[3,4-d]pyrimidin-4-one (10b). Mp 160-161 ${ }^{\circ} \mathrm{C}$ (crystallized from methanol); yield: 79\%; IR ( $\mathrm{cm}^{-1}$ ): 3067 (CH aromatic); 2928 (CH aliphatic); $1709(\mathrm{C}=\mathrm{O}) ; 1596(\mathrm{C}=\mathrm{N}) ;{ }^{1} \mathrm{H}$ NMR (DMSO-d $d_{6} \delta$ ppm: 2.10 (s, 3H); $2.41(\mathrm{~s}, 3 \mathrm{H})$; 7.38-7.40 (m, 1H); 7.52-7.57 (m, 4H); 7.99-8.01 (m, 4H); $10.94(\mathrm{~s}, 1 \mathrm{H})$; Anal. Calcd for $\mathrm{C}_{20} \mathrm{H}_{16} \mathrm{FN}_{5} \mathrm{O}$ (361.38): C, 66.47; H, 4.46; N, 19.38. Found: C, 66.38; H, 4.02; N, 19.61.

(E)-3,6-Dimethyl-5-(4-methoxybenzylideneamino)-1-phenyl-1,5-dihydropyrazolo[3,4-d]pyrimidin-4one (10c). Mp 255-256 ${ }^{\circ} \mathrm{C}$ (crystallized from toluene); yield: 55\%; IR ( $\left.\mathrm{cm}^{-1}\right)$ : 3027 (CH aromatic); $1671(\mathrm{C}=\mathrm{O}) ; 1594(\mathrm{C}=\mathrm{N}) ;{ }^{1} \mathrm{H}-\mathrm{NMR}\left(\mathrm{DMSO}-d_{6}\right) \delta \mathrm{ppm}: 2.49(\mathrm{~s}, 3 \mathrm{H}) ; 2.51(\mathrm{~s}, 3 \mathrm{H}) ; 3.84(\mathrm{~s}, 3 \mathrm{H}) ; 7.22$ $(\mathrm{t}, J=6 \mathrm{~Hz}, 1 \mathrm{H}) ; 7.37-7.57(\mathrm{~m}, 6 \mathrm{H}) ; 8.07(\mathrm{~d}, J=9 \mathrm{~Hz}, 2 \mathrm{H}) ; 8.85(\mathrm{~s}, 1 \mathrm{H}) ; \mathrm{MS} m / z: 373$ $\left(\mathrm{M}^{+}, 10.42\right)$; Anal. Calcd for $\mathrm{C}_{21} \mathrm{H}_{19} \mathrm{~N}_{5} \mathrm{O}_{2}$ (373.42): C, 67.55; H, 5.13; N, 18.75. Found: C, 67.10; $\mathrm{H}$, $5.01 ; \mathrm{N}, 18.47$.

(E)-5-(4-Chlorobenzylideneamino)-3,6-dimethyl-1-phenyl-1,5-dihydropyrazolo[3,4-d]pyrimidin-4-one (10d). $\mathrm{Mp}>300{ }^{\circ} \mathrm{C}$ (crystallized from ethanol); yield: 60\%; IR $\left(\mathrm{cm}^{-1}\right): 3064(\mathrm{CH}$ aromatic); $2925(\mathrm{CH}$ aliphatic); $1698(\mathrm{C}=\mathrm{O}) ; 1596(\mathrm{C}=\mathrm{N}) ;{ }^{1} \mathrm{H}-\mathrm{NMR}\left(\mathrm{DMSO}-d_{6}\right) \delta$ ppm: 2.09 (s, 3H); $2.54(\mathrm{~s}, 3 \mathrm{H}) ; 7.37$ (t, $J=8.1 \mathrm{~Hz}) ; 7.56(\mathrm{t}, J=8.1 \mathrm{~Hz}, 2 \mathrm{H}) ; 7.66(\mathrm{~d}, J=8.4 \mathrm{~Hz}, 2 \mathrm{H}) ; 7.99$ (d, $J=8.4 \mathrm{~Hz}, 2 \mathrm{H}) ; 8.32$ $(\mathrm{d}, J=8.7 \mathrm{~Hz}, 2 \mathrm{H}) ; 8.32(\mathrm{~s}, 1 \mathrm{H}) ;{ }^{13} \mathrm{C}-\mathrm{NMR}\left(\mathrm{DMSO}-d_{6}\right) \delta \mathrm{ppm}: 13.72,23.32,104.40,121.72,127.13$, 129.68, 129.83, 130.93, 131.63, 137.96, 138.66, 146.86, 150.30, 154.86, 157.74, 168.91. MS m/z: 377 $\left(\mathrm{M}^{+}, 11.12\right)$, Anal. Calcd for $\mathrm{C}_{20} \mathrm{H}_{16} \mathrm{ClN}_{5} \mathrm{O}$ (377.85): C, 63.58; H, 4.27; N, 18.51. Found: C, 63.28; H, $4.04 ; \mathrm{N}, 18.13$.

(E)-3,6-Dimethyl-5-(4-nitrobenzylideneamino)-1-phenyl-1,5-dihydropyrazolo[3,4-d]pyrimidin-4-one (10e). (Crystallized from acetic acid); $\mathrm{mp}>300{ }^{\circ} \mathrm{C}$; yield: $95 \%$; IR $\left(\mathrm{cm}^{-1}\right)$ : 3057 (CH aromatic); 2925 (CH aliphatic); $1690(\mathrm{C}=\mathrm{O}) ; 1597(\mathrm{C}=\mathrm{N}) ;{ }^{1} \mathrm{H}-\mathrm{NMR}\left(\mathrm{DMSO}-d_{6}\right) \delta$ ppm: $2.60(\mathrm{~s}, 3 \mathrm{H}) ; 2.61(\mathrm{~s}, 3 \mathrm{H})$; 7.39 (t, $J=7.8 \mathrm{~Hz}, 1 \mathrm{H}) ; 7.57$ (t, $J=7.8 \mathrm{~Hz}, 2 \mathrm{H}) ; 8.07$ (d, $J=8.7 \mathrm{~Hz}, 2 \mathrm{H}) ; 8.25(\mathrm{~d}, J=9 \mathrm{~Hz}, 2 \mathrm{H}) ; 8.42$ (d, $J=9 \mathrm{~Hz}, 2 \mathrm{H}) ; 9.16(\mathrm{~s}, 1 \mathrm{H})$; $\mathrm{MS} m / z: 388\left(\mathrm{M}^{+}, 18.10\right)$. Anal. Calcd for $\mathrm{C}_{20} \mathrm{H}_{16} \mathrm{~N}_{6} \mathrm{O}_{3}$ (388.39): C, 61.85; H, 4.15; N, 21.64. Found: C, 62.15; H, 4.44; N, 21.40. 


\subsection{Biological Testing}

\subsubsection{Materials and Methods}

Human breast cancer cell line, MCF was grown as monolayer culture in RPM 11640 medium supplemented with $10 \%$ fetal bovine serum (FBS) and 1\% penicillin/streptomycin. The cell line was incubated at $37{ }^{\circ} \mathrm{C} 5 \% \mathrm{CO}_{2} 95 \%$ air and high humidity atmosphere in the water jacketed incubator (Revco, GS Laboratory Equipment, RCO 3000 TVBB, Asheville, NC, USA). The cell line was regularly subcultured to be maintained in the exponential growth phase. The sterile conditions were strictly attained by working under the equipped laminar flow (Microflow laminar flow cabinet, Hamsphire SP 105aa, Andover, UK).

\subsubsection{Measutement of Potential Cytotoxicity}

The cytotoxicity was carried out using the sulphorhodamine-B (SRB) assay. Cells were seeded in 96 well microtiter plates at a concentration of 1,000-2,000 cells/well, $100 \mu \mathrm{L} /$ well, After $24 \mathrm{~h}$, cells will be incubated for $72 \mathrm{~h}$ with various concentrations of drugs $(0,0.01,0.1,1,10$ and $100 \mu \mathrm{g} / \mathrm{mL})$. For each derivative concentration and doxorubicin, 3 wells were used. The plates were incubated for $72 \mathrm{~h}$. The medium is discarded. The cells were fixed with $150 \mu \mathrm{L}$ cold trichloroacetic acid $10 \%$ final concentration for $1 \mathrm{~h}$ at $4{ }^{\circ} \mathrm{C}$.

The plates were washed with distilled water using a Tecan automatic washer (Crailsheim, Germany) and stained with $50 \mu \mathrm{L} 0.4 \%$ SRB dissolved in $1 \%$ acetic acid for $30 \mathrm{~min}$ at room temperature in dark. The plates were washed with $1 \%$ acetic acid to remove unbound dye and air-dried $(24 \mathrm{~h})$.

The dye was solubilized with $150 \mu \mathrm{L} /$ well of $10 \mathrm{mMtris}$ base (PH 7.4) for $5 \mathrm{~min}$ on a shaker at $1,600 \mathrm{rpm}$. The optical density (OD) of each well will be measured spectrophotometrically at $490 \mathrm{~nm}$ with an ELISA microplate reader. The mean background absorbance was automatically subtracted and mean values of each derivative and doxorubicin concentration was calculated. The experiment was repeated 3 times. The percentage of cell survival was calculated as follows:

$$
\text { Surviving fraction }=\text { O.D. }(\text { treated cells }) / \text { O.D. }(\text { control cells })
$$

\section{Conclusions}

In conclusion, series of new 5-substituted pyrazolo[3,4- $d$ ]pyrimidin-4-ones 4, 5a,b, 6, 7, 8a-e, 10a-e were synthesized. The cytotoxic activity of the newly synthesized compounds against the human breast adenocarcinoma cell line MCF-7 was investigated. Almost all the tested compounds revealed some antitumor activity, in particular 3,6-dimethyl-5-(4-nitrobenzylideneamino)-1-phenyl-1,5-dihydropyrazolo $[3,4-d]$ pyrimidin-4-one (10e) which exhibited the highest activity among the tested compounds with an $\mathrm{IC}_{50}$ equal to $11 \mu \mathrm{M}$. The antitumor screening revealed that aromatic substitution on the N5 position favors the activity. Moreover, 5-(4-substituted benzylideneamino) derivatives 10a-e with azomethine spacer groups were more potent than the 5-(4-substituted phenyl) derivatives $\mathbf{8 a}-\mathbf{e}$. 


\section{Acknowledgments}

We are grateful to Samia Shouman, of Pharmacology, and all members of the Department of Cancer Biology, National Cancer Institute, Cairo, Egypt, for their kind help on carrying out the cytotoxicity testing.

\section{Author Contributions}

K.R.A.A. proposed the subject, designed the study and offered necessary guidance to R.B.B to carry out the synthesis, purification and characterization of the compounds. E.K.A.A. and M.A.A. conceived the study and participated in its design, results and discussion, and coordination. R.R.A. carried out the biological assay.

\section{Conflicts of Interest}

The authors declare no conflict of interest.

\section{References}

1. Wu, H.; Chang, D.; Huang, C. Targeted therapy for cancer. J. Cancer Mol. 2006, 2, 57-66.

2. Sierra, J.R.; Cepero, V.; Giordano, S. Molecular mechanisms of acquired resistance to tyrosine kinase targeted therapy. Mol. Cancer 2010, 9, 75-88.

3. Riley, L.B.; Desai, D.C. The molecular basis of cancer and the development of targeted therapy. Surg. Clin. N. Am. 2009, 89, 1-15.

4. Cheng, C.C.; Robins, R.K. Potential Purine Antagonists. VI. Synthesis of 1-alkyl- and 1-aryl-4substituted pyrazolo[3,4-d]pyrimidines. J. Org. Chem. 1956, 21, 1240-1256.

5. Ismail, Z.H.; Abdel-Gawad, S.M.; Abdel-Aziem, A.; Ghorab, M.M. Synthesis of some new biologically active sulfur compounds containing pyrazolo[3,4-d]pyrimidine moiety. Phosphor. Sulfur Silicon 2003, 178, 1795-1805.

6. Carraro, F.; Naldini, A.; Pucci, A.; Locatelli, G.A.; Maga, G.; Schenone, S.; Bruno, O.; Ranise, A.; Bondavalli, F.; Brullo, C.; et al. Pyrazolo[3,4-d]pyrimidines as potent antiproliferative and proapoptotic agents toward A431 and 8701-BC cells in culture via inhibition of c-Src phosphorylation. J. Med. Chem. 2006, 49, 1549-1561.

7. El-Enany, M.M.; Kamel, M.M.; Khalil, O.M.; El-Nassan, H.B. Synthesis and antitumor activity of novel 6-aryl and 6-alkylpyrazolo[3,4-d]pyrimidin-4-one derivatives. Eur. J. Med. Chem. 2010, $45,5286-5291$.

8. Abd El Hamid, M.K.; Mihovilovic, M.D.; El-Nassan, H.B. Synthesis of novel pyrazolo[3,4d]pyrimidine derivatives as potential anti-breast cancer agents. Eur. J. Med. Chem. 2012, 57, 323-328.

9. Kandeel, M.M.; Mohamed, L.W.; Abd El Hamid, M.K.; Negmeldin, A.T. Design, synthesis, and antitumor evaluation of novel pyrazolo[3,4-d]pyrimidine derivatives. Sci. Pharm. 2012, 80, 531-545.

10. Peat, A.J.; Garrido, D.; Boucheron, J.A.; Schweiker, S.L.; Dickerson, S.H.; Wilson, J.R.; Wang, T.Y.; Thomson, S.A. Novel GSK-3 inhibitors with improved cellular activity. Bioorg. Med. Chem. Lett. 2004, 14, 2127-2130. 
11. Kim, D.C.; Lee, Y.R.; Yang, B.S.; Shin, K.J.; Kim, D.J.; Chung, B.Y.; Yoo, K.H. Synthesis and biological evaluations of pyrazolo[3,4-d]pyrimidines as cyclin-dependent kinase 2 inhibitors. Eur. J. Med. Chem. 2003, 38, 525-532.

12. Schenone, S.; Brullo, C.; Bruno, O.; Bondavalli, F.; Mosti, L.; Maga, G.; Crespan, E.; Carraro, F.; Manetti, F.; Tintori, C.; et al. Synthesis, biological evaluation and docking studies of 4-amino substituted 1H-pyrazolo[3,4-d]pyrimidines. Eur. J. Med. Chem. 2008, 43, 2665-2676.

13. Schenone, S.; Bruno, O.; Bondavalli, F.; Ranise, A.; Mosti, L.; Menozzi, G.; Fossa, P.; Manetti, F.; Morbidelli, L.; Trincavelli, L.; et al. 1-(2-Chloro-2-phenylethyl)6-methylthio-1H-pyrazolo[3,4d]pyrimidines-4-amino substituted and their biological evaluation. Eur. J. Med. Chem. 2004, 39, 153-160.

14. Ghorab, M.M.; Ragab, F.A.; Alqasoumi, S.I.; Alafeefy, A.M.; Aboulmagd, S.A. Synthesis of some new pyrazolo[3,4-d]pyrimidine derivatives of expected anticancer and radioprotective activity. Eur. J. Med. Chem. 2010, 45, 171-178.

15. Hassan, G.S.; Kadry, H.H.; Abou-Seri, S.M.; Ali, M.M.; Mahmoud, A.E. Synthesis and in vitro cytotoxic activity of novel pyrazolo[3,4-d]pyrimidines and related pyrazole hydrazones toward breast adenocarcinoma MCF-7 cell line. Bioorg. Med. Chem. 2011, 19, 6808-6817.

16. Reck, M.; Mok, T.; Wolf, J.; Heigener, D.; Wu, Y.L. Reviewing the safety of erlotinib in non small cell lung cancer. Expert Opin. Drug Saf. 2011, 10, 147-157.

17. Omar, H.A.; Sargeant, A.M.; Weng, J.-R.; Wang, D.; Kulp, S.K.; Patel, T.; Chen, C.-S. Pulmonary endothelial impairment during gefitinib therapy: A preliminary assessment with iodine-123metaiodobenzylguanidine (1231-MIBG) scintigraphy. Open Lung Cancer J. 2011, 4, 957-968.

18. Fang, L.; Barekati, Z.; Zhang, B.; Liu, Z.; Zhong, X. Targeted therapy in breast cancer: What's new? Eur. J. Med. Sci. 2011, 141, 1-9.

19. Bakr, R.B.; Abdelall, E.K.A.; Abdel-Hamid, M.K.; Kandeel, M.M. Design and synthesis of new EGFR-tyrosine kinase inhibitors containing pyrazolo[3,4-d]pyrimidine cores as anticancer agents. Bull. Pharm. Sci. Assiut Univ. 2012, 35, 1-16.

20. Heravi, M.M.; Nami, N.; Seifi, N.; Oskooie, H.A.; Hekmatshoar, R. Microwave-assisted synthesis of substituted pyrazoles and pyrazolo[3,4-d]thiopyrimidines. Phosphor. Sulfur Silicon 2006, 181, 591-599.

21. Vichai, V.; Kirtikara, K. Sulforhodamine B colorimetric assay for cytotoxicity screening. Nat. Protoc. 2006, 1, 1112-1116.

Sample Availability: Samples of the compounds 1-10 are available from the authors.

(C) 2014 by the authors; licensee MDPI, Basel, Switzerland. This article is an open access article distributed under the terms and conditions of the Creative Commons Attribution license (http://creativecommons.org/licenses/by/3.0/). 